\title{
3 Research Square

\section{Ipa1 Improves Rice Drought Tolerance at Seedling Stage Mainly Through Activating Abscisic Acid Pathway}

\section{Menghao Zhu}

Wuhan University

Yonggang $\mathrm{He}$

Wuhan University

Mingqiang Zhu

Wuhan University

Ayaz Ahmad

Wuhan University

Shuang Xu

Wuhan University

\section{Zijun He}

Wuhan University

Shan Jiang

Wuhan University

Jinqiu Huang

Wuhan University

Zhihui Li

Wuhan University

Shaojia Liu

Wuhan University

Xin Hou

Wuhan University

Zhihong Zhang ( $\sim$ zzh@whu.edu.cn )

Wuhan University https://orcid.org/0000-0002-1063-1186

\section{Research Article}

Keywords: Rice (Oryza sativa L.), Drought Tolerance, ipa1, Carbon-Nitrogen Metabolism, Abscisic Acid (ABA)

Posted Date: July 27th, 2021 
DOI: https://doi.org/10.21203/rs.3.rs-733123/v1

License: (c) (1) This work is licensed under a Creative Commons Attribution 4.0 International License. Read Full License

Version of Record: A version of this preprint was published at Plant Cell Reports on October 25th, 2021. See the published version at https://doi.org/10.1007/s00299-021-02804-3. 


\section{Abstract}

Drought is a major abiotic stress to crop production. IPA1 (IDEAL PLANT ARCHITECTURE 1)/OSSPL14 encodes a transcription factor and has been reported to function in both rice ideal plant architecture and biotic resistance. Here, with a pair of IPA1/ipa 1-NILs (Near Iso-genic Lines), we found that ipa 1 could significantly improve rice drought tolerance at seedling stage. The ipa 1 plants had a better-developed root system and smaller leaf stomatal aperture. Analysis of carbon-nitrogen metabolism-associated enzyme activity, gene expression, and metabolic profile indicated that ipa 1 could tip the carbon-nitrogen metabolism balance towards an increased carbon metabolism pattern. In both the control and PEGtreated conditions, ABA content in the ipa 1 seedlings was significantly higher than that in the IPA1 seedlings. Expression of the ABA biosynthesis genes was detected to be up-regulated, whereas the expression of ABA catabolism genes was down-regulated in the ipa 1 seedlings. In addition, based on yeast one-hybrid assay and dual-luciferase assay, IPA1 was found to directly activate the promoter activity of OsHOX12, a transcription factor promoting ABA biosynthesis, and OsNAC52, a positive regulator of the ABA pathway. The expression of OsHOX12 and OSNAC52 was significantly up-regulated in the ipa 1 plants. Combined with the previous studies, our results suggested that ipa 1 could improve rice seedling drought tolerance mainly through activating the ABA pathway and that regulation of the ipa 1mediated ABA pawthway will be an important strategy for improving drought resistance of rice.

\section{Key Messages}

ipa1 enhances rice drought tolerance mainly through activating the ABA pathway. It endows rice seedlings with a more developed root system, smaller leaf stomata aperture, and enhanced carbon metabolism.

\section{Introduction}

Plants live in fixed locations and face diverse abiotic stresses (such as drought, salinity, and cold) negatively affecting plant growth and seed production. To survive, plants have evolved high plasticity and complex mechanisms to respond to these stimuli over a long period of time (Hu and Xiong 2014). The understanding of plant responses to stresses in physiology, genetics, and molecular biology will be greatly helpful in improving the tolerance of plants to abiotic stresses through genetic engineering (Huang, et al. 2009).

Metabolic adaption to abiotic stress is important to plant surviving under unfavorable conditions(Barnaby, et al. 2019; Ma, et al. 2016). Generally, nitrogen promotes plant shoot growth rather than root, while carbon does oppositely, and high carbon/nitrogen $(\mathrm{C} / \mathrm{N})$ ratio enhances plant root development with a high root/shoot ratio (Osuna, et al. 2015), which allows plant root access to water profoundly and decreases shoot water losses. Accumulation of carbohydrates resulting from enhanced photosynthesis protects plants from membrane damage and accounts, in part, for the more vigorous growth during stress (Garg, et al. 2002). On the contrary, increasing nitrogen levels increased the degree 
of water stress, resulting in decreased leaf water potential, especially when the total water applied was minimal (Aragon and De Datta 1982). Moreover, high C/N status may act as a stress condition, which induces a series of stress-related genes, including transcription factors such as OSMYB4, CHS (key enzyme in flavonoid biosynthesis) and genes involved in the jasmonate signaling pathway (Huang, et al. 2016).

Abscisic acid ( $A B A)$ is a multifunctional plant hormone that regulates many physiological processes, including seed dormancy and germination, stomatal movement, and plant responses to abiotic stress. NCED (9-cis-epoxycarotenoid dioxygenase) is the key rate-limiting enzyme in ABA biosynthetic pathway. Overexpression of OSNCED3 in Arabidopsis results in increased accumulation of ABA, reduced relative water loss, delayed seed germination, and greater drought tolerance relative to that of wild-type (Hwang, et al. 2010). Rice nced3 mutants had increased sensitivity to water and $\mathrm{H}_{2} \mathrm{O}_{2}$ stress, increased stomata aperture, delayed leaf senescence, and decreased ABA content, while overexpression of OsNCED 3 could enhance rice water stress tolerance, promote leaf senescence and increase ABA content (Huang, et al. 2018). ABA 8'-hydroxylase is considered as the main ABA catabolic enzyme. OsABA80x3 RNAi lines showed significant improvement in drought stress tolerance with increased ABA content. In contrast, overexpression seedlings were hypersensitive to drought stress with decreased $A B A$ content, indicating OsABA80x3 gene plays an important role in controlling $A B A$ level and drought stress resistance in rice (Cai, et al. 2015).

Stress response at the molecular level involves induction of stress-responsive and stress-tolerant genes. Many transcription factors have been identified to be involved in plant adaptation to abiotic stresses (Baillo, et al. 2019). SQUAMOSA PROMOTER BINDING PROTEIN-LIKE (SPL) family transcription factors sharing a highly conserved SBP domain are plant-specific, and their functions are surprisingly diverse, covering virtually every aspect of plant growth and development and response to stresses (Wang and Wang 2015). In rice genome, about $19 S P L$ genes have been identified. Among these, as a multifunctional gene in regulating plant development, IPA1/OSSPL14 has attracted extensive attention. It was reported firstly to function in rice "Ideal Plant Architecture (IPA)" characterized by fewer unproductive tillers, larger panicles and stronger culms (Jiao, et al. 2010; Miura, et al. 2010). Since then, IPA1 was also identified to play a vital role in rice biotic resistance (Liu, et al. 2019; Wang, et al. 2018). Overexpressing of IPA1 could enhance rice resistance to Xanthomonas oryzae $p v$. oryzae, partially through gibberellin signaling, including interacting with SLR1 and enhancing GA metabolism by activating EUl1 expression (Liu, et al. 2019).

Although great progress has been made in understanding the roles of IPA1 in rice plant development and biotic resistance, its function in rice abiotic stresse tolerance is still unknown. Here, by using a pair of the IPA1/ipa 1-NILs (Near Iso-genic Lines), we found that ipa1 could significantly improve rice drought tolerance at the seedling stage mainly through activating ABA pathway.

\section{Materials And Methods}




\section{Plant materials}

A pair of the ipa1/IPA1-NILs was developed from a cross of an indica cv. Yuetai B (IPA1/IPA1) and a japonica cv. Shaoniejing (SNJ) (ipa1/ipa1). Yuetai B was crossed with SNJ to develop $\mathrm{F}_{1}$ plants, then the $F_{1}$ plants were backcrossed to Yuetai $B$ to develop $B_{1} F_{1}$ plants. The $B_{1} F_{1}$ plants were self-crossed for six generations to develop $\mathrm{F}_{7}$ plants, from which a plant with a genotype IPA1/ipa1 was identified. Let this plant self-cross and, from its offspring, the plants with genotype IPA1/IPA1 were identified as a IPA1$\mathrm{NIL}$, while the plants with genotype ipa1/ipa1 as a ipa 1-NIL. The degree of genomic similarity for the NILs is $95.8 \%$. The ipa 1 plants showed fewer tillers and leaves, but more panicle branches ( Supplementary table 1), as reported previously (Jiao, et al. 2010).

\section{Hydroponic culture conditions}

Seeds were disinfected in $20 \%$ sodium hypochlorite solution for $30 \mathrm{~min}$, thoroughly washed with deionized water. Sterilized seeds were germinated in distilled water for $48-72 \mathrm{~h}$ at $30^{\circ} \mathrm{C}$ in darkness and then transferred to hydroponic culture solution as described previously (Wang, et al. 2018). Fresh solution was changed every 3 days, and $\mathrm{pH}$ was adjusted to 5.5 every day.

\section{Drought stress and osmotic stress experiments}

In soil drought experiments, two-leaf stage seedlings cultured in sandy soil were treated with dehydration by removing water with PVC pipes for 7 days and then re-watered for 5 days. For PEG treatment, two-leaf stage seedlings were transferred to culture solution containing 25\% (w/v) PEG4000 for 5 days, and then recovered for 4 days.

\section{Imaging of rice leaf stomata}

Imaging of rice leaf stomata was conducted as described previously (Wang, et al. 2016) with some modifications. Leaves of 15 days seedlings under control and $6 \mathrm{~h} 25 \%$ PEG treatment conditions were immediately fixed by $2.5 \%$ glutaraldehyde, and stomatal pictures were obtained by scanning electron microscopy (JSM-6390LV, JEOL, Tokyo, Japan).

\section{Carbon-nitrogen metabolism-associated indexes measurement}

After cultured for $20,28,36,44$ and 52 days in nutrient solution under natural conditions, the leaves of IPA1/ipa 1 seedlings were collected for carbon-nitrogen metabolism-associated indexes measurement. Soluble sugar and sucrose contents were determined using the anthrone method (Shields and Burnett 1960) and the resorcinol method (Han, et al. 2015), respectively. FBP (Fructose 1,6-bisphosphatase) activity was determined according to kit instructions (Solarbio, Beijing, China). PEPC (Phosphopyruvate carboxylase) activity was measured by the previously method (BLANKE and EBERT 1992). SPS (Sucrose Phosphate Synthase) and SS (Sucrose Synthase) activities were measured as described previously (Shi, et al. 2016). 
Soluble protein and free amino acid contents were determined using the Bradford assay (Bradford 1976) and the ninhydrin method (Sun, et al. 2006), respectively. Nitrate content was measured as described previously (Doane and Horwáth 2003). NR (Nitrate reductase), GS (Glutamine synthase), GOGAT (Glutamate synthase), and GDH (Glutamate dehydrogenase) activities were estimated based on the previously methods (Li, et al. 2016).

\section{Metabolomics analysis}

The shoot bases of IPA1/ipa 1 seedlings grown for 21 days in nutrient solution under natural conditions were collected for metabolomics analysis. The sample preparation, extract analysis, metabolite identification and quantification were performed as described previously (Chen, et al. 2014) at Wuhan Metware Biotechnology Co., Ltd., Wuhan, China.

\section{Phytohormone measurement}

Leaves of 15 days seedlings under control and 6 h 25\% PEG treatment conditions were collected for phytohormone measurement. Plant materials were ground into powder in liquid nitrogen, and extracted with $80 \%$ methanol at $4^{\circ} \mathrm{C}$. The extract was centrifuged at $12,000 \mathrm{~g}$ under $4^{\circ} \mathrm{C}$ for $15 \mathrm{~min}$. The supernatant was collected and evaporated to dryness under nitrogen gas stream, and then reconstituted in $30 \%$ methanol. The solution was centrifuged, and the supernatant was collected for LC-MS analysis. The LC-MS analysis was conducted with the API6500 QTRAP LC/MS/MS system, equipped with an ESI Turbo lon-Spray interface, operating in a positive ion mode and controlled by Analyst 1.6 software (AB Sciex).

\section{RNA extraction and real-time PCR}

After treated with $25 \%$ PEG for $0,3,6$ and $9 \mathrm{~h}$, leaves of 15 days seedlings were collected for RNA extraction. Total RNA was extracted with TRIzol (Invitrogen, Carlsbad, CA, USA) reagent. According to the manufacturer, RNA sample $(\sim 2 \mu \mathrm{g})$ was treated with DNasel and then used for CDNA synthesis with the Super-Script III first-strand cDNA synthesis system (Invitrogen, Carlsbad, CA, USA). Real-time PCR was performed using 2×SYBR Green PCR Master Mix (Takara, Dalian, China) in a CFX96TM Real-Time System (BIO-RAD, Hercules, CA, USA). Each experiment included three technical replicates and three biological replicates. OsActin was used as an internal control for normalization. Primers used are listed in Supplementary Table 2.

\section{Dual-luciferase assays}

The promoters of OsHOX12 and OsNAC52 were amplified by PCR from genomic DNA and cloned into pGreenll 0800-LUC reporter vectors in front of luciferase (LUC) gene. Besides, the Renilla luciferase (REN) reporter gene was driven by the CaMV 35S promoter as a control in each transformation. The coding region of IPA1 was cloned behind the Ubi promoter into the effector vector pRGV (He, et al. 2018). The reporter and effector were transformed into in rice protoplasts. The luciferase activities were measured using the Dual-Luciferase Reporter Assay System (Promega, Beijing, China) and compared with empty vector-transformed plants. 


\section{Yeast one-hybrid assay}

The coding region of IPA $1_{S B P}$ was amplified and cloned into the $\mathrm{PGADT7}$ prey vector. The promoters of OsHOX12 and OsNAC52 were amplified and cloned into pAbAi bait vectors. The prey vectors, respectively, co-transformed with bait vector into Y1HGOLD strain. These transformed cells were grown on SD/-Leu/Ura plates and then grown on SD/-Leu/-Ura/ AbAi plates at $28^{\circ} \mathrm{C}$ for $3-5$ days.

\section{Results}

ipa1 enhances rice drought tolerance at seedling stage

To investigate the effect of ipa 1 on rice drought tolerance, a soil drought experiment was performed with a pair of the IPA1/ipa 1-NILs. The ipa1-NIL obtained a survival rate of $83.5 \%$, while it was only $20.8 \%$ for the IPA1-NIL (Fig. 1a, c). As treated with 25\% PEG4000 (osmotic stress simulating drought stress), the ipa1 seedlings exhibited a survival rate of $62.1 \%$, while the IPA1-NIL showed a significantly lower survival rate of $43.5 \%$ (Fig. $1 \mathrm{~b}, \mathrm{~d}$ ). These results indicated that ipa 1 could significantly improve rice drought tolerance at seedling stage.

\section{The characteristics of root and leaf stomata for the IPA1/ipa1 seedlings}

As compared to that of the IPA1 seedlings, the ipa1 seedlings had significantly increased root length, root and shoot dry weight as well as the dry weight ratio of root to shoot (Fig. 2a-e).

As for leaf stomata, the ipa 1 seedlings showed a decreased stoma size as compared with that of the IPA1 seedlings (Fig. 2f, g), despite no significant difference in stoma density between the NILs (Supplementary Fig. 1). Moreover, the ipa 1 plants displayed more stomata completely close (29.3\%) and less stomata completely open (18.5\%) than that of the IPAT-NIL (14.0\% and $34.7 \%$, respectively). When treated with PEG, more leaf stomata tended to close for both the IPA1 and ipa 1 seedlings. Even so, there were still more stomata completely close $(56.6 \%$ ) and less stomata completely open (nearly $0.0 \%$ ) for the ipa 1 seedlings than that for the IPA 1 seedlings ( $45.9 \%$ and $8.3 \%$, respectively) (Fig. $2 \mathrm{~h}$ ). Consequently, the ipa1 seedlings were found to lose less water (Fig. 2i).

The ipa 1 plants had a better developed root system conducive to enhancing their ability to absorb water from the soil and their leaves with smaller stomatal aperture were beneficial to enhance their moisturizing function, which could play a vital physiological role in improving their drought tolerance.

The ipa 1 seedling could adjust its carbon-nitrogen metabolism balance to a metabolic pattern with a relatively strong carbon metabolism

It was found that the ipa 1 plants had a higher content for soluble sugar and sucrose than the IPA1 plants in both leaves (Fig. 3a, b) and sheaths (Supplementary Fig. 2). Then, we measured the activity of several 
carbon metabolism-related enzymes. Except for FBP, three carbon metabolism-related enzymes (PEPC, SPS, and SS) showed a activity level higher in the ipa 1 plants than in the IPA1 plants (Fig. 3c-f).

As for the nitrogen metabolism, soluble protein and free amino acid contents for the ipa 1 seedlings were significantly lower than that for the IPA1 plants (Fig. 4a, b). Meanwhile, the ipa 1 plants were found to be significantly higher in inorganic nitrate-nitrogen content than the IPA1 plants (Fig. 4c). Four key nitrogen assimilation enzymes (NR, GS, GOGAT and GDH) were investigated and each of them showed a significantly lower activity level in the ipa 1 plants than in the IPA1 plants (Fig. 4d-g). Accordingly, the expression of genes involved in nitrogen absorption, transport and assimilation was detected to be downregulated, especially in the roots of the ipa 1 plants (Supplementary Fig. 3).

Combined with the above findings, it seems that the ipa 1 plant could change its carbon-nitrogen metabolism balance to a metabolic pattern with a relatively stronger carbon metabolism by enhancing its carbon metabolic activity and down-regulating its nitrogen metabolism, thus benefiting the accumulation of carbohydrates in the plant, which could provide a stronger material and energy basis for the plants to tolerate external abiotic stress.

\section{Metabolic profile analysis of the IPA1/ipa1 plants}

To further explore the effect of ipa 1 on plant metabolism, we analyzed the metabolic profiles with the IPA1/ipa 1-NILs by using a liquid chromatography-electrospray ionization-tandem mass spectrometry. There were 357 compounds to be identified. These compounds covered the key components involved in metabolic pathways of sugars, amino acids, nucleotide, organic acids, fatty acids and others (Supplementary Table 3).

The majority of carbohydrates such as sucrose, trehalose 6-phosphate (T6P), glucosamine and glucarate o-phosphoric acid were detected to accumulate more in the ipa 1 plants than in the IPA1 plants, except glucose (Fig. 5a). For the key metabolites involved in nitrogen assimilation, the ipa1 plants showed a significantly decrease in the contents of amino acids Tyr and Trp (Fig. 5b) while their precursor shikimic acid mainly accumulated in the ipa 1 plants (Fig. 5c). Similarly, the levels of organic acids (2-OG, succinic acid, and malic acid) and amino acids (Gln, Glu, Asn and Asp, as the major forms of nitrogen in xylem sap of rice plant) were significantly decreased in the ipa1 plants (Fig. 5b, c) while their precursor aconitic acid (a major element in TCA cycle) also showed to accumulate significantly in the ipa 1 plants (Fig. 5c). These results indicated that the carbon flux to nitrogenous compounds was depressed in the ipa 1 plants as compared to the IPA1 plants. Besides that, most of the other identified amino acids, amino acid derivates and nucleotides were decreased to different degrees in the ipa 1 plants (Supplementary Fig. 4a, b). Therefore, all these results suggested that the gene ipa 1 could significantly influence the balance of carbon-nitrogen metabolism, tipping the carbon/nitrogen metabolism balance towards increased carbon metabolism.

Cysteine is the first carbon/nitrogen-reduced sulfur product resulting from the sulfate assimilation pathway. As a sulfur donor, it plays a major role in the growth and development of plant. Glutathione 
derived from cysteine protects plants from reactive oxygen species (ROS) damage caused by abiotic stress (Droux 2004). In this study, a significantly increased cysteine content was found in the ipa1 plants, coupled with an increase of reduced glutathione content and a decrease of oxidized glutathione content (Fig. 5d). Moreover, contents of the other antioxidants such as coumarin and curcumin raised dramatically in the ipa 1 plants (Supplementary Fig. 4c). The same situation also happened to glycerophospholipids (Supplementary Fig. 4d), the cell membrane major components. Therefore, ipa 1 may activate sulfate assimilation and the related defense mechanism, which plays an essential role in protecting the ipa 1 plants from ROS damage under abiotic stresses. In addition, ferulic acid is reported to be a marker metabolite for plant drought resistance and high photosynthesis ( $\mathrm{Ma}$, et al. 2016). The contents of ferulic acid-related metabolites were significantly upregulated in the ipa 1 seedlings (Supplementary Fig. 4e).

\section{The enhanced drought tolerance in the ipa1 plants could be mediated by ABA accumulation}

ABA and GAs are known to be primary phytohormones that antagonistically regulate plant abiotic stress resistance (Vishal and Kumar 2018). In this study, exogenous ABA application led to an obvious inhibition on plant height, whereas $\mathrm{GA}_{3}$ treatment significantly promoted the trait for both the NILs under nondrought stress conditions (Supplementary Fig. 5). When PEG was applied to simulate drought conditions (osmotic stress), the ABA application significantly improved survival rates of seedlings, whereas $\mathrm{GA}_{3}$ decreased survival rates for both the NILs (Fig. 6a, b). Although so, the two NILs showed significant differences in degree of response to $\mathrm{ABA}$ and $\mathrm{GA}_{3}$ treatments. Comparatively, the ipa 1 seedlings were less sensitive to $A B A$ or $\mathrm{GA}_{3}$ treatment. Exogenous $A B A$ treatment improved PEG resistance of the IPA1 plants to a greater extent, resulting in a fact that survival rate of the IPA1 plants was no longer different from that for the ipa 1 plants (Fig. 6a, b).

Then, we measured ABA and GAs contents of the NILs. In both control and PEG-treated conditions, ABA content in the ipa 1 seedlings was significantly higher than that in the IPA1 seedlings (Fig. 6c). Meanwhile, the ABA biosynthesis genes such as OsNCED1, OsNCED3, and OsNCED4 were detected to be up-regulated, whereas ABA catabolism genes were down-regulated in ipa 1-NIL (Fig. 6e). OsABI5, OsLEA3, OsLIP9, and OsRAB16A are marker genes of the ABA pathway involved in abiotic stress response (Zhang, et al. 2015). In our study, all these marker genes were up-regulated significantly in the ipa1 plants under the control and PEG-treatment conditions (Fig. 6f).

As for GAs, with an exception of a remarkable increase of the $\mathrm{GA}_{4}$ content in ipa 1-NIL under the control condition, no significant difference has been observed in the contents of GAs investigated between the two NILs under the control or PEG-treated conditions (Fig. 6d), although several genes for GA biosynthesis and catabolism showed some differences in expression levels between the two NILs (Supplementary Fig. 6).

The above results suggested that the enhanced drought tolerance of the ipa1-NIL could mainly result from a high level of ABA accumulation in the ipa 1 seedlings. 
IPA1 directly activated the expression of OsHOX12 and OsNAC52

OsHOX12 is a transcription factor homologous with AtHB21, 40 and 53. It was reported to activate expression of OsNCED1, and promote ABA biosynthesis in rice (Liu, et al. 2020). OsNAC52, a transcription factor belonging to NAC family, potentially responds to ABA and confers drought tolerance in transgenic plants (Gao, et al. 2010). In addition, as a transcription activator, IPA1 can regulate its target gene by directly binding to the core motif GTAC or indirectly to the core motif TGGGCC/T of the target gene promoter (Lu, et al. 2013). Bioinformatics analysis identified twelve and three GTAC motifs in the promoters of OsHOX12 and OsNAC52, respectively (Fig. 7a, b). We searched previously published ChIPseq data of IPA1 (Lu, et al. 2013), and found that OsHOX12 and OsNAC52 were potential targets of IPA1, suggesting that IPA1 may directly activate the expression of OsHOX12 and OsNAC52.

To test the hypothesis, we conducted a yeast one-hybrid assay. Cells co-transformed with bait vectors and prey vectors grew well on SD/-Leu/-Ura/AbAi plates, indicating that IPA1 can directly bind to the promoters of OsHOX12 and OsNAC52 (Fig. 7c, d). Then, we carried out a dual-luciferase assay using the full length of the OsHOX12 and OsNAC52 promoters in rice protoplasts. Co-transformed reporter vectors and effector vectors activated the expression of $\angle U C$ gene, suggesting that IPA1 can significantly enhance the activity of the OsHOX12 and OsNAC52 promoters (Fig. 7e, f). Accordingly, the expression of OsHOX12 was increased in the ipa1 seedlings under PEG-treated conditions (Fig. 7h). Moreover, the expression of OsNAC52 was also significantly up-regulated in the ipa 1 plants under both the control and PEG-treated conditions (Fig. 7i).

We also tested expression of the other genes involved in abiotic stresses in the NIL plants. OsNAC5, OsNAC6, and OsNAC19 are three other NAC family transcription factors, and overexpression of each of those was reported to enhance rice resistance to abiotic stresses (Hu, et al. 2006; Takasaki, et al. 2010). The results depicted that, these genes' expression showed a significantly higher level in the ipa 1 plants than in the IPA1 plants under control and PEG-treated conditions (Fig. 7j-I).

\section{Discussion}

IPA1/OSSPL 14 is one of the most concerned genes in current studies of rice functional genomics due to its multifunctions in regulating plant development (Wang, et al. 2018). In this study, we found that ipa1 could significantly improve rice drought tolerance at seedling stage. The ipa1 seedlings demonstrated a better-developed root system in terms of phenotypes, which helped to enhance their ability to absorb water from the soil. Their leaves with smaller stomatal aperture improved their moisturizing ability, which could play a crucial physiological role in enhancing their resistance to drought tolerance.

$A B A$ is induced in response to adverse environmental conditions, and it plays a critical role in regulating abiotic stress response in plants (Cutler, et al. 2010). Deficit of ABA in nced3 mutants increased rice sensitivity to water stress, while accumulation of ABA in OSNCED3-overexpressing seedings enhanced rice water stress tolerance (Huang, et al. 2018). In this study, the ipa1 seedlings had a significantly higher ABA content than the IPA1 plants (Fig. 6c). The ABA pathway marker genes were up-regulated by ipa 1 
(Fig. 6e). Exogenous ABA treatment largely promoted PEG resistance for the IPA1 plants (Fig. 6a, b). These results seem to suggest that the improved drough tolerance might result from activation of the ABA pathway in the ipa1 seedlings.

OsHOX12 and OsNAC52 are two of the transcription factors involved in ABA pathway in rice (Gao, et al. 2010; Liu, et al. 2020). Our yeast one-hybrid assay and dual-luciferase test indicated that IPA1 can directly bind to the promoters of OsHOX12 and OsNAC52 (Fig. 7c, d) and significantly enhance the activity of the OsHOX12 and OsNAC52 promoters. Therefore, IPA1 may directly activate OsHOX12, thus promoting ABA biosynthesis. Meanwhile, our study indicated that ipa 1 could enhance the ABA pathway by directly regulating OsNAC52, which was reported to be a positive regulator of the ABA pathway (Gao, et al. 2010). Additionally, Gonzalez-Grandio et al. (2017) reported that the TCP (TEOSINTE BRANCHED1, CYCLOIDEA, PCF) transcription factor BRANCHED1 (BRC1) in Arabidopsis binds to and positively regulates the transcription of three related Homeodomain leucine zipper protein (HD-ZIP) encoding genes HOMEOBOX PROTEIN 21 (HB21), HOMEOBOX PROTEIN 40 (HB40), and HOMEOBOX PROTEIN 53 (HB53), which together with BRC1, enhances 9-CIS-EPOXICAROTENOID DIOXIGENASE 3 (NCED3) expression, leading to ABA accumulation and triggering hormone response. In rice, Lu et al. (2013) revealed that IPA1 directly targets OSTB1 (an ortholog of BRC1). Here, we showed that OSTB1 was up-regulated significantly in the ipa1 seedlings (Supplementary Fig. 7). Therefore, we speculate that, as in Arabidopsis, IPA1 could also target OsTB1 to enhance the expression of the ABA biosynthesis genes, thus leading to ABA accumulation in ipa 1 seedlings.

ABA is also a key regulator of plant stomatal aperture and root development. In response to drought stress, plants can synthesize $A B A$, which triggers closing of stomatal pores, thus reducing water loss (Schroeder, et al. 2001). The foliage-derived ABA promoted root growth relative to shoot growth but inhibited the development of lateral roots (McAdam, et al. 2016). Moreover, a very recent paper showed that moderate enhancement of ABA signaling helps maintain the RM (root meristem) size, sustaining root growth by antagonizing the GA-promoted degradation of OsSHR1 through the SnRK2-APC/CTE regulatory module, while mutants of OSABA1 (a ABA biosynthesis gene) displayed a short root phenotype (Lin, et al. 2020). Therefore, the smaller stomatal and better-developed root system in ipa 1 seedlings could result from the accumulation of ABA.

The ABA pathway also plays a vital role in regulating plant metabolism. Treatment with low level ABA increases rice soluble sugar and decreases free amino acid contents (Zeng, et al. 2009). SnRK2s are key positive regulators of ABA signaling. Overexpression of SnRK2.6 promotes plant carbon assimilation with drastically boosted sucrose and total soluble sugar levels in the leaves through increasing SPS activity (Zheng, et al. 2010). Moreover, srk2d srk2e srk2i (snrk2.2/2.3/2.6) showed enhanced TCA cycle (key source of C-skeletons for amino acid synthesis), leading to a higher carbon flux into amino acids and potentially proteins under non-stress conditions (Yoshida, et al. 2019). Our results indicated that ipa 1 plants could adjust the balance of carbon-nitrogen metabolism by enhancing their carbon metabolic activity, but relatively down-regulating their nitrogen metabolism (Fig. 3,4). Metabolic profile analysis further supported such a change of carbon-nitrogen metabolism in ipa1 plants (Fig. 5a, b). Thus, for the 
ipa 1 seedlings, tipping the carbon/nitrogen balance towards increased carbon metabolism might also result from the enhancement of $A B A$ signaling, which contributes to their enhanced drought tolerance. Further detailed analyses are required to understand the interaction of $\mathrm{ABA}$ pathway and $\mathrm{C} / \mathrm{N}$ metabolism balance in the ipa 1 plants.

It should be noted that, the results of the current study revealed the effect of the gene ipa1 on drought tolerance of rice plants only at seedling stage. At this stage, OSTB1 is the most important target of IPA1, and this target gene is mainly expressed at seedling stage (Lu, et al. 2013). As rice plants develop into panicle differentiation stage, the main targets of IPA1 turn into genes such as OSDEP1 (Lu, et al. 2013). Accordingly, rice plants' hormone regulation and metabolic pattern could be changed remarkably, which is worthy of further study in the future. Additionally, the gene ipa 1 is one of the most essential yieldincreasing genes reported in rice so far (Wang, et al. 2018; Yu, et al. 2020). It increases rice yield mainly by shaping ideal plant type with fewer tillers and larger panicles (Jiao, et al. 2010). In this study, the ipa 1 seedlings were observed to have a larger dry weight per plant (Fig. 2c, d), although their nitrogen metabolism was down-regulated relative to its WT seedlings. Obviously, the ipa 1 rice plants with larger biomass at the seedling stage would be more likely to develop larger panicles later, thus contributing to increased yields.

In conclusion, this study elucidated that ipa 1 could significantly improve rice drought tolerance at seedling stage. The ipa 1 plants had a better-developed root system and smaller leaf stomatal aperture. They could tip the carbon-nitrogen metabolism balance towards an increased carbon metabolism pattern. Meanwhile, the ABA biosynthesis genes were up-regulated, whereas the ABA catabolism genes were down-regulated in the ipa1 seedlings, resulting in accumulation of endogenous $A B A$. Based on yeast one-hybrid assay and dual-luciferase assay, IPA1 was found to directly activate the expression of OsHOX12 and OsNAC52, a transcription factor promoting ABA biosynthesis and a positive regulator of the ABA pathway, respectively. These results suggested that ipa 1 could improve rice seedling drought tolerance mainly through activating the ABA pathway, and that it may has potential applications in improving drought resistance of rice.

\section{Declarations}

\section{Acknowledgements}

The authors thank Dr. Q.Qian of the China National Rice Research Institute for providing the ipa1 donor rice $\mathrm{cv}$. Shaoniejing used in this particular research. This work was financed by the Special Transgenic Program of the Ministry of Agriculture in China (No. 2016ZX08001004-002), the National Natural Science Foundation of China (No.31901522), and the Collaborative Innovation Center of Hubei Province for Hybrid Rice.

Author contribution statement $M Z$ and $Z Z$ designed the experiments. $M Z$ performed most experiments. $\mathrm{MZ}, \mathrm{YH}$ and $\mathrm{ZZ}$ analyzed the data. MZ, AA, SX, ZH, SJ, JH, ZL and SL assisted in materials and data collection. MZ, XH and ZZ drafted the manuscript. 


\section{Compliance with ethical standards}

Conflict of interest The authors declare that they have no confict of interest.

\section{References}

1. Aragon EL, De Datta SK (1982) Drought response of rice at different nitrogen levels using line source sprinkler system. Irrig Sci 3:63-73

2. Baillo EH, Kimotho RN, Zhang Z, Xu P (2019) Transcription factors associated with abiotic and biotic stress tolerance and their potential for crops improvement. Genes (Basel) 10:771-793

3. Barnaby JY, Rohila JS, Henry CG, Sicher RC, Reddy VR, McClung AM (2019) Physiological and metabolic responses of rice to reduced soil moisture: relationship of water stress tolerance and grain production. Int J Mol Sci 20:1846

4. BLANKE MM, EBERT G (1992) Phosphoenolpyruvate carboxylase and carbon economy of apple seedlings. J Exp Bot 43:965-968

5. Bradford MM (1976) A rapid and sensitive method for the quantitation of microgram quantities of protein utilizing the principle of protein-dye binding. Anal Biochem 72:248-254

6. Cai S, Jiang G, Ye N, Chu Z, Xu X, Zhang J, Zhu G (2015) A key ABA catabolic gene, OsABA8ox3, is involved in drought stress resistance in rice. PLoS One 10:e0116646

7. Chen W, Gao Y, Xie W, Gong L, Lu K, Wang W, Li Y, Liu X, Zhang H, Dong H, Zhang W, Zhang L, Yu S, Wang G, Lian X, Luo J (2014) Genome-wide association analyses provide genetic and biochemical insights into natural variation in rice metabolism. Nat Genet 46:714-721

8. Cutler SR, Rodriguez PL, Finkelstein RR, Abrams SR (2010) Abscisic acid: emergence of a core signaling network. Annu Rev Plant Biol 61:651-679

9. Doane TA, Horwáth WR (2003) Spectrophotometric determination of nitrate with a single reagent. Anal Lett 36:2713-2722

10. Droux M (2004) Sulfur assimilation and the role of sulfur in plant metabolism: a survey. Photosynth Res 79:331-348

11. Gao F, Xiong A, Peng R, Jin X, Xu J, Zhu B, Chen J, Yao Q (2010) OsNAC52, a rice NAC transcription factor, potentially responds to $A B A$ and confers drought tolerance in transgenic plants. Plant Cell Tiss Organ Cult 100:255-262

12. Garg AK, Kim JK, Owens TG, Ranwala AP, Choi YD, Kochian LV, Wu RJ (2002) Trehalose accumulation in rice plants confers high tolerance levels to different abiotic stresses. Proc Natl Acad Sci USA 99:15898-15903

13. Gonzalez-Grandio E, Pajoro A, Franco-Zorrilla JM, Tarancon C, Immink RG, Cubas P (2017) Abscisic acid signaling is controlled by a BRANCHED1/HD-ZIP / cascade in Arabidopsis axillary buds. Proc Natl Acad Sci USA 114(2):E245-E254 
14. Han H, Tian Z, Fan Y, Cui Y, Cai J, Jiang D, Cao W, Dai T (2015) Water-deficit treatment followed by rewatering stimulates seminal root growth associated with hormone balance and photosynthesis in wheat (Triticum aestivum L.) seedlings. Plant Growth Regul 77:201-210

15. He F, Zhang F, Sun W, Ning Y, Wang GL (2018) A versatile vector toolkit for functional analysis of rice genes. Rice (N Y) 11:27

16. Hu H, Dai M, Yao J, Xiao B, Li X, Zhang Q, Xiong L (2006) Overexpressing a NAM, ATAF, and CUC (NAC) transcription factor enhances drought resistance and salt tolerance in rice. Proc Natl Acad Sci USA 103:12987-12992

17. Hu H, Xiong L (2014) Genetic engineering and breeding of drought-resistant crops. Annu Rev Plant Biol 65:715-741

18. Huang A, Sang Y, Sun W, Fu Y, Yang Z (2016) Transcriptomic analysis of responses to imbalanced carbon: nitrogen availabilities in rice seedlings. PLoS One 11:e0165732

19. Huang XY, Chao DY, Gao JP, Zhu MZ, Shi M, Lin HX (2009) A previously unknown zinc finger protein, DST, regulates drought and salt tolerance in rice via stomatal aperture control. Genes Dev 23:18051817

20. Huang Y, Guo Y, Liu Y, Zhang F, Wang Z, Wang H, Wang F, Li D, Mao D, Luan S, Liang M, Chen L (2018) 9-cis-Epoxycarotenoid Dioxygenase 3 regulates plant growth and enhances multi-abiotic stress tolerance in rice. Front Plant Sci 9:162

21. Hwang SG, Chen HC, Huang WY, Chu YC, Shii CT, Cheng WH (2010) Ectopic expression of rice OSNCED3 in Arabidopsis increases ABA level and alters leaf morphology. Plant Sci 178:12-22

22. Jiao Y, Wang Y, Xue D, Wang J, Yan M, Liu G, Dong G, Zeng D, Lu Z, Zhu X, Qian Q, Li J (2010) Regulation of OSSPL 14 by OsmiR156 defines ideal plant architecture in rice. Nat Genet 42:541-544

23. Li H, Liang Z, Ding G, Shi L, Xu F, Cai H (2016) A natural light/dark cycle regulation of carbon-nitrogen metabolism and gene expression in rice shoots. Front Plant Sci 7:1318

24. Lin Q, Zhang Z, Wu F, Feng M, Sun Y, Chen W, Cheng Z, Zhang X, Ren Y, Lei C, Zhu S, Wang J, Zhao Z, Guo X, Wang H, Wan J (2020) The APC/C(TE) E3 ubiquitin ligase complex mediates the antagonistic regulation of root growth and tillering by ABA and GA. Plant Cell 32:1973-1987

25. Liu M, Shi Z, Zhang X, Wang M, Zhang L, Zheng K, Liu J, Hu X, Di C, Qian Q, He Z, Yang DL (2019) Inducible overexpression of Ideal Plant Architecture1 improves both yield and disease resistance in rice. Nat Plants 5:389-400

26. Liu X, Hu Q, Yan J, Sun K, Liang Y, Jia M, Meng X, Fang S, Wang Y, Jing Y, Liu G, Wu D, Chu C, Smith SM, Chu J, Wang Y, Li J, Wang B (2020) Z-carotene isomerase suppresses tillering in rice through the coordinated biosynthesis of strigolactone and abscisic acid. Mol Plant 13:1784-1801

27. Lu Z, Yu H, Xiong G, Wang J, Jiao Y, Liu G, Jing Y, Meng X, Hu X, Qian Q, Fu X, Wang Y, Li J (2013) Genome-wide binding analysis of the transcription activator IDEAL PLANT ARCHITECTURE1 reveals a complex network regulating rice plant architecture. Plant Cell 25:3743-3759

28. Ma X, Xia H, Liu Y, Wei H, Zheng X, Song C, Chen L, Liu H, Luo L (2016) Transcriptomic and metabolomic studies disclose key metabolism pathways contributing to well-maintained 
photosynthesis under the drought and the consequent drought-tolerance in rice. Front Plant Sci 7:1886

29. McAdam SA, Brodribb TJ, Ross JJ (2016) Shoot-derived abscisic acid promotes root growth. Plant Cell Environ 39:652-659

30. Miura K, Ikeda M, Matsubara A, Song XJ, Ito M, Asano K, Matsuoka M, Kitano H, Ashikari M (2010) OSSPL 14 promotes panicle branching and higher grain productivity in rice. Nat Genet 42:545-549

31. Osuna D, Prieto P, Aguilar M (2015) Control of seed germination and plant development by carbon and nitrogen availability. Front Plant Sci 6:1023

32. Schroeder JI, Kwak JM, Allen GJ (2001) Guard cell abscisic acid signalling and engineering drought hardiness in plants. Nature 410:327-330

33. Shi H, Wang B, Yang P, Li Y, Miao F (2016) Differences in sugar accumulation and mobilization between sequential and non-sequential senescence wheat cultivars under natural and drought conditions. PLoS One 11:e0166155

34. Shields R, Burnett W (1960) Determination of protein-bound carbohydrate in serum by modified anthrone method. Anal Chem 32:885-886

35. Sun S-W, Lin Y-C, Weng Y-M, Chen M-J (2006) Efficiency improvements on ninhydrin method for amino acid quantification. J Food Compos Anal 19:112-117

36. Takasaki H, Maruyama K, Kidokoro S, Ito Y, Fujita Y, Shinozaki K, Yamaguchi-Shinozaki K, Nakashima K (2010) The abiotic stress-responsive NAC-type transcription factor OsNAC5 regulates stress-inducible genes and stress tolerance in rice. Mol Genet Genomics 284:173-183

37. Vishal B, Kumar PP (2018) Regulation of seed germination and abiotic stresses by gibberellins and abscisic acid. Front Plant Sci 9:838

38. Wang B, Smith SM, Li J (2018) Genetic regulation of shoot architecture. Annu Rev Plant Biol 69:437468

39. Wang H, Wang H (2015) The miR156/SPL module, a regulatory hub and versatile toolbox, gears up crops for enhanced agronomic traits. Mol Plant 8:677-688

40. Wang J, Zhou L, Shi H, Chern M, Yu H, Yi H, He M, Yin J, Zhu X, Li Y, Li W, Liu J, Wang J, Chen X, Qing H, Wang Y, Liu G, Wang W, Li P, Wu X, Zhu L, Zhou J-M, Ronald PC, Li S, Li J, Chen X (2018) A single transcription factor promotes both yield and immunity in rice. science 361:1026-1028

41. Wang L, Yu C, Xu S, Zhu Y, Huang W (2016) OsDi19-4 acts downstream of OsCDPK14 to positively regulate ABA response in rice. Plant Cell Environ 39:2740-2753

42. Wang Q, Nian J, Xie X, Yu H, Zhang J, Bai J, Dong G, Hu J, Bai B, Chen L, Xie Q, Feng J, Yang X, Peng J, Chen F, Qian Q, Li J, Zuo J (2018) Genetic variations in ARE1 mediate grain yield by modulating nitrogen utilization in rice. Nat Commun 9:735

43. Yoshida T, Obata T, Feil R, Lunn JE, Fujita Y, Yamaguchi-Shinozaki K, Fernie AR (2019) The role of abscisic acid signaling in maintaining the metabolic balance required for Arabidopsis growth under nonstress conditions. Plant Cell 31:84-105 
44. Yu S, Ali J, Zhang C, Li Z, Zhang Q (2020) Genomic breeding of green super rice varieties and their deployment in Asia and Africa. Theor Appl Genet 133:1427-1442

45. Zeng Z, Yi Z, Wang G, Wang Y (2009) Influence of seed soaking with ABA on the physiological and yield-contributing traits of rice seedlings. J Southwest Univ (Nat Sci Ed) 31:52-56

46. Zhang DP, Zhou Y, Yin JF, Yan XJ, Lin S, Xu WF, Baluska F, Wang YP, Xia YJ, Liang GH, Liang JS (2015) Rice G-protein subunits QPE9-1 and RGB1 play distinct roles in abscisic acid responses and drought adaptation. J Exp Bot 66:6371-6384

47. Zheng Z, Xu X, Crosley RA, Greenwalt SA, Sun Y, Blakeslee B, Wang L, Ni W, Sopko MS, Yao C, Yau K, Burton S, Zhuang M, McCaskill DG, Gachotte D, Thompson M, Greene TW (2010) The protein kinase SnRK2.6 mediates the regulation of sucrose metabolism and plant growth in Arabidopsis. Plant physiol 153:99-113

\section{Supplementry}

Supplementary Table 1 is not available with this version.

\section{Figures}

a
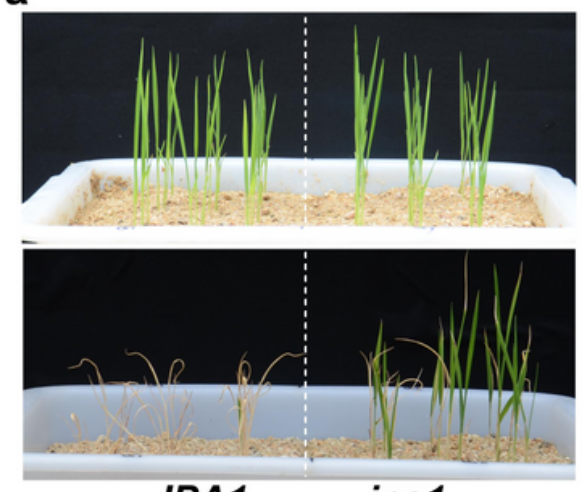

IPA1 ipa1 b

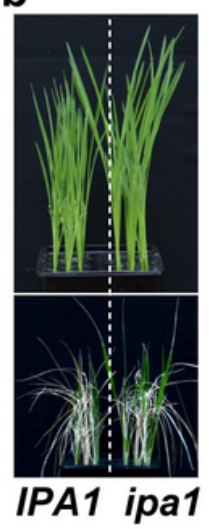

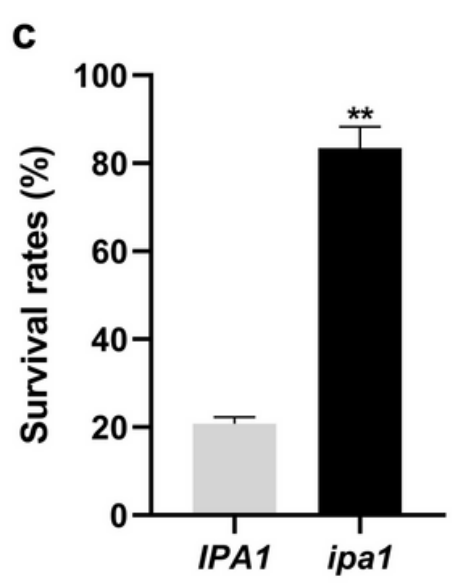

d

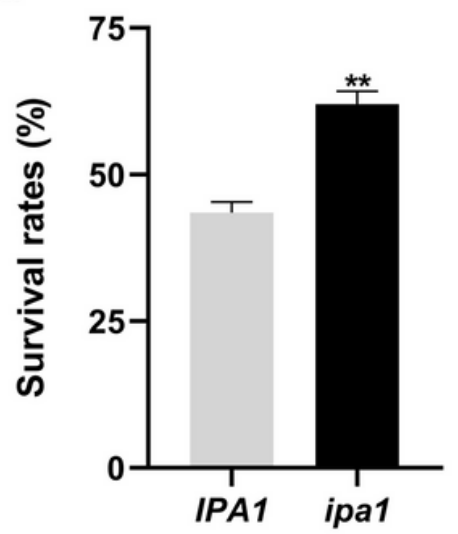

\section{Figure 1}

Drought tolerance of the IPA1/ipa1 seedlings. $(a, b)$ Performance of the IPA1/ipa1 seedlings under soil drought and $25 \%$ PEG4000 treatments, respectively. (c, d) Survival rates of the IPA1/ipa1 seedlings treated with soil drought and $25 \%$ PEG4000, respectively. Each experiment has three replicates, and twenty-four (c) or thirty-six (d) seedlings were tested in each replicate. Data represent the means $\pm \mathrm{SE}$. ${ }^{*} \mathrm{P}<0.05$, t-test. ${ }^{*} \mathrm{P}<0.01$, t-test. 
a
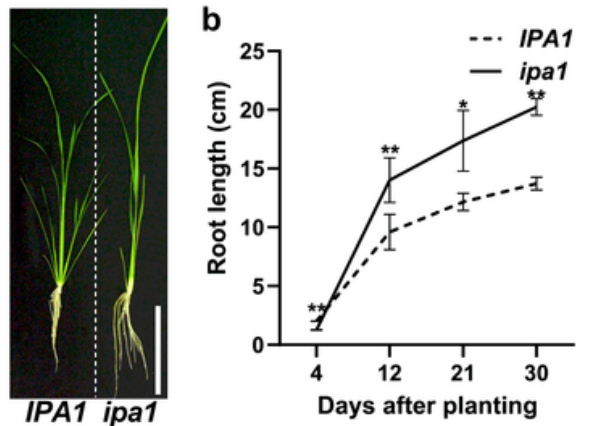

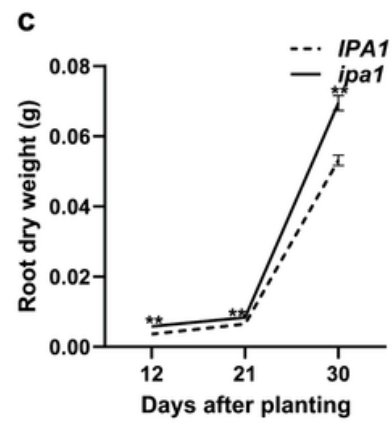

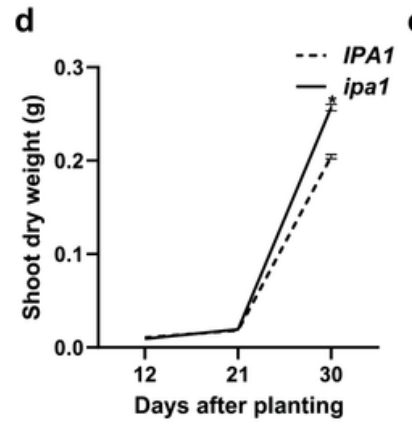
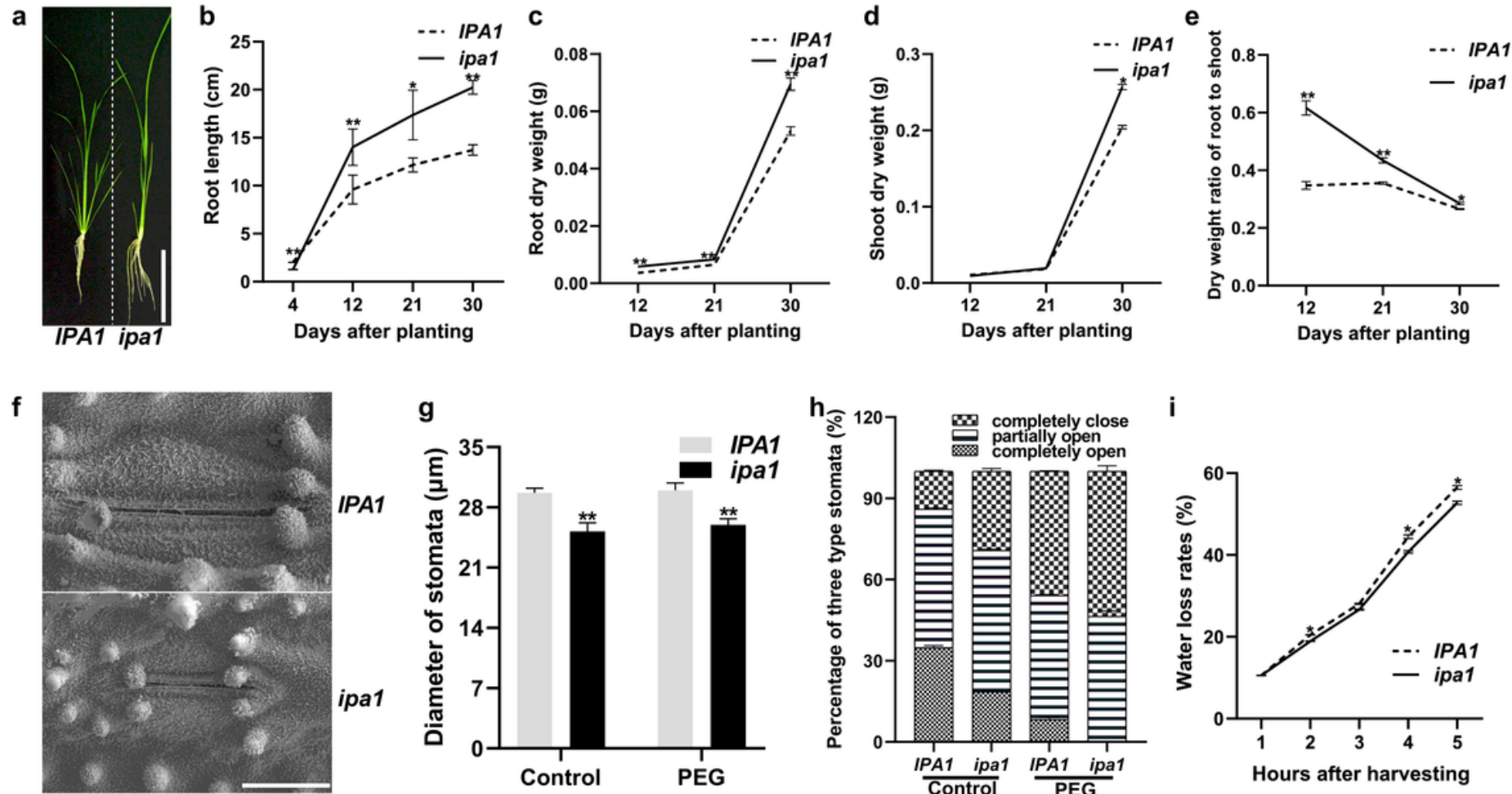

i

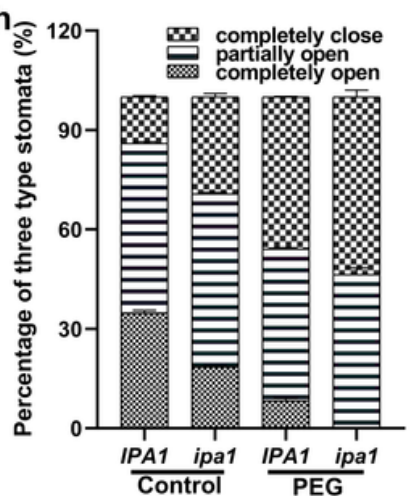

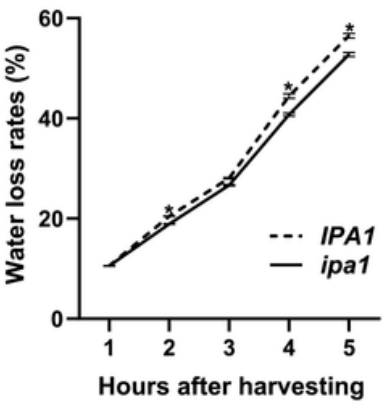

Figure 2

Characteristics of root and leaf stomata for the IPA1/ipa1 seedlings. (a) Plant architecture of the IPA1/ipa1 seedlings twenty-five days after planting. Bar, $10 \mathrm{~cm}$. (b-e) Root length, Root dry weight, Shoot dry weight and Dry weight ratio of root to shoot, respectively. b-e, $n=18$. (f) Scanning electron microscopy (SEM) images of IPA1/ipa1 seedlings. Bar, $10 \mu \mathrm{m}$. (g) The stomatal diameter $(\mathrm{n}=12$, derived from three seedlings). (h) Percentage of three types of stomata. Forty-seven to fifty-two stomata from three seedlings were calculated. (i) The water loss rates, $n=15$. PEG, 25\% PEG4000 treatment condition. Data represent the means $\pm S E$. ${ }^{*} P<0.05$, t-test. ${ }^{*}{ }^{*} \mathrm{P}<0.01$, $t$-test. 


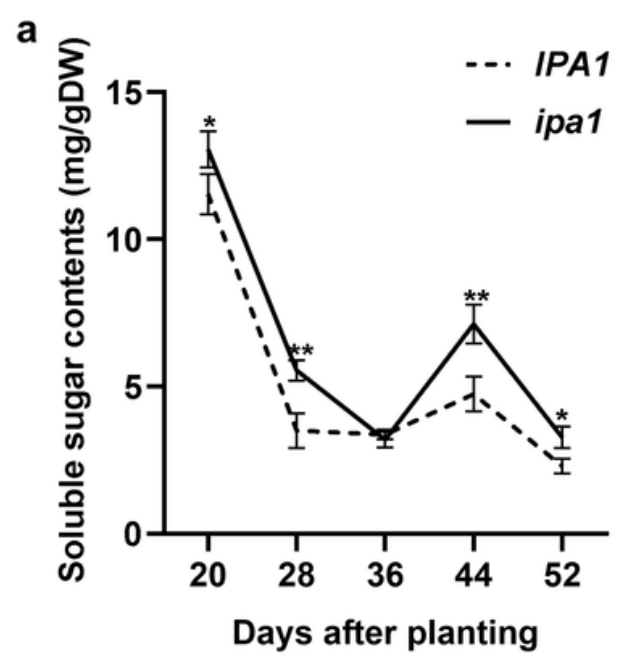

d

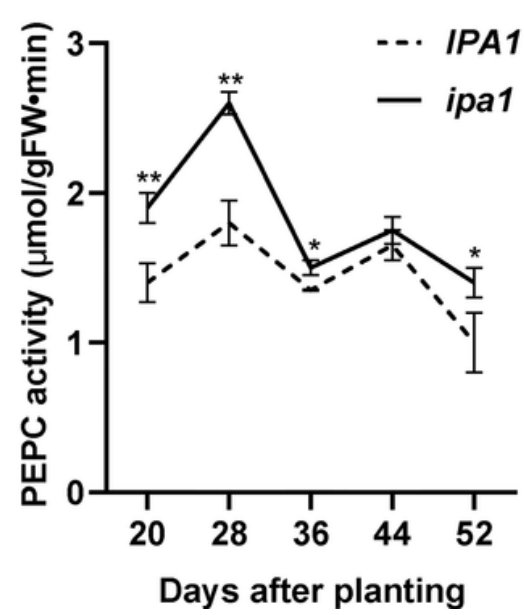

b

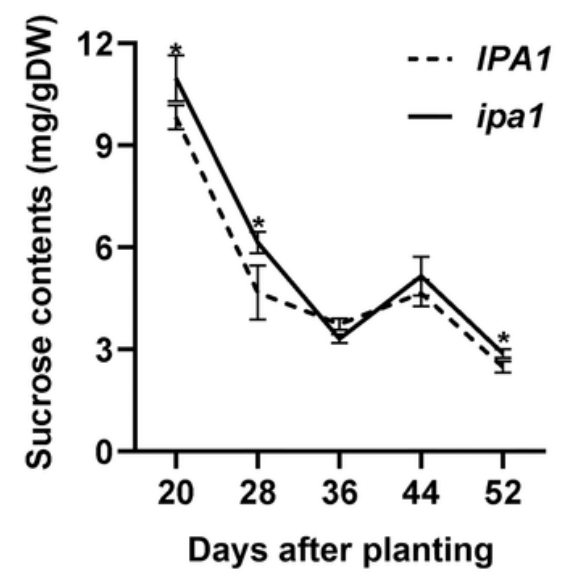

e

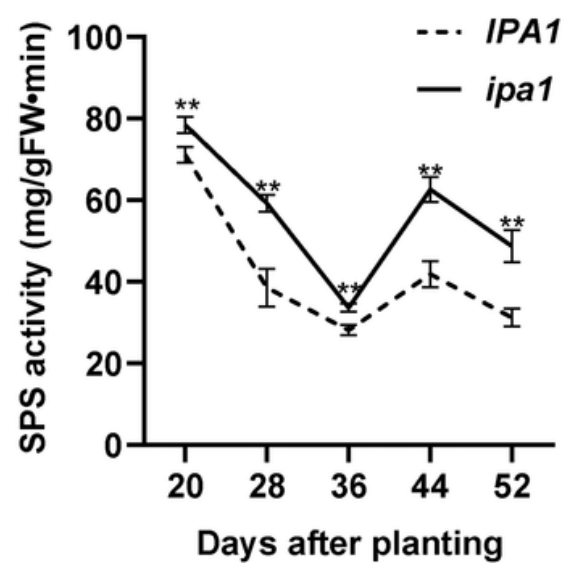

C

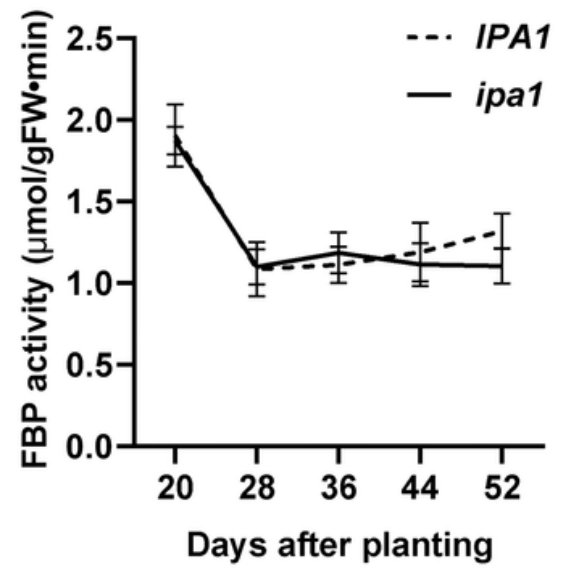

f

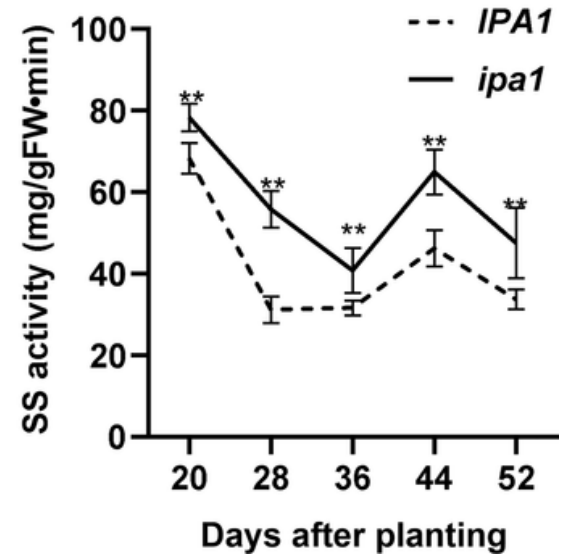

Figure 3

Carbon metabolism-associated indices of the IPA1/ipa1 seedlings. (a, b) Contents of soluble sugar and sucrose, respectively, in the IPA1/ipa1 seedling leaves. (c-f) Activity of FBP, PEPC, SPS and SS, respectively, in the IPA1/ipa1 seedlings. Data represent the means $\pm S E(n=3) .{ }^{*} P<0.05$, $t$-test. ${ }^{* *} P<0.01$, $t$ test. 
a

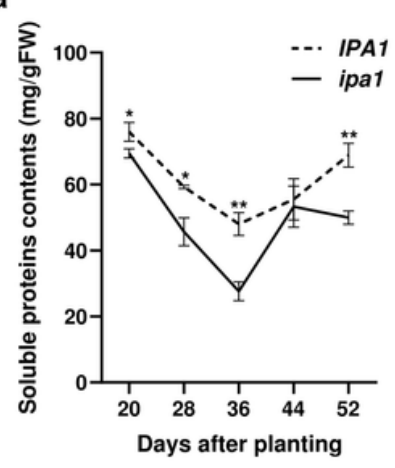

b

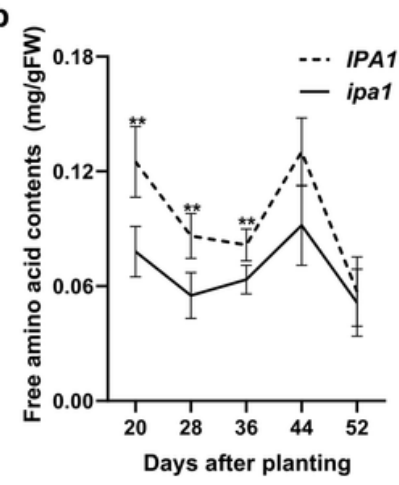

e

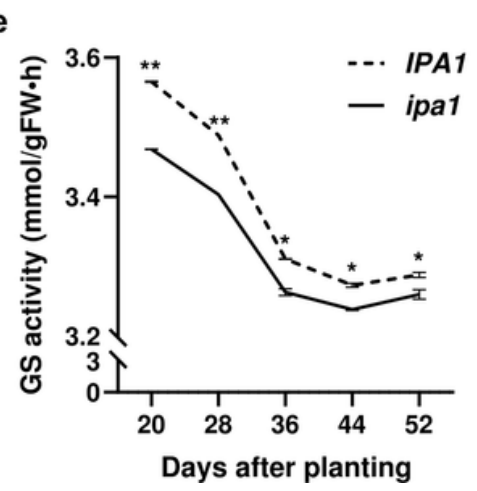

c

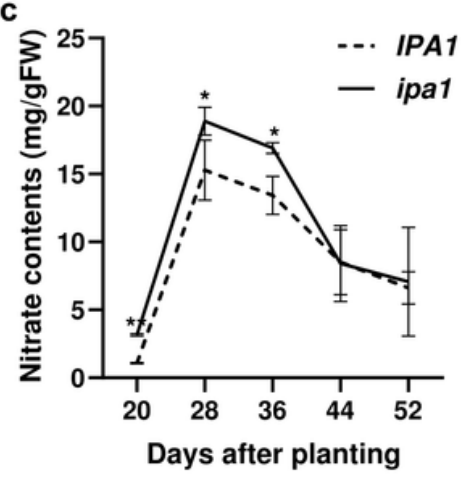

g

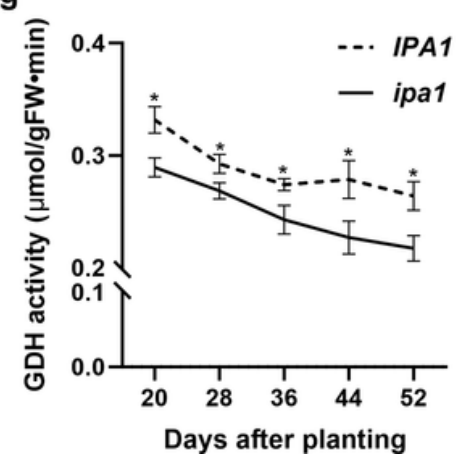

Figure 4

Nitrogen metabolism-associated indices of the IPA1/ipa1 seedlings. (a-c) Contents of soluble proteins, free amino acid and nitrate, respectively, in leaves of the IPA1/ipa1 seedlings. (d-g) Activity of NR, GS, GOGAT and GDH, respectively, in the IPA1/ipa1 seedlings. Data represent the means $\pm S E(n=3) .{ }^{*}<<0.05$, t-test. ${ }^{*} \mathrm{P}<0.01$, t-test. 

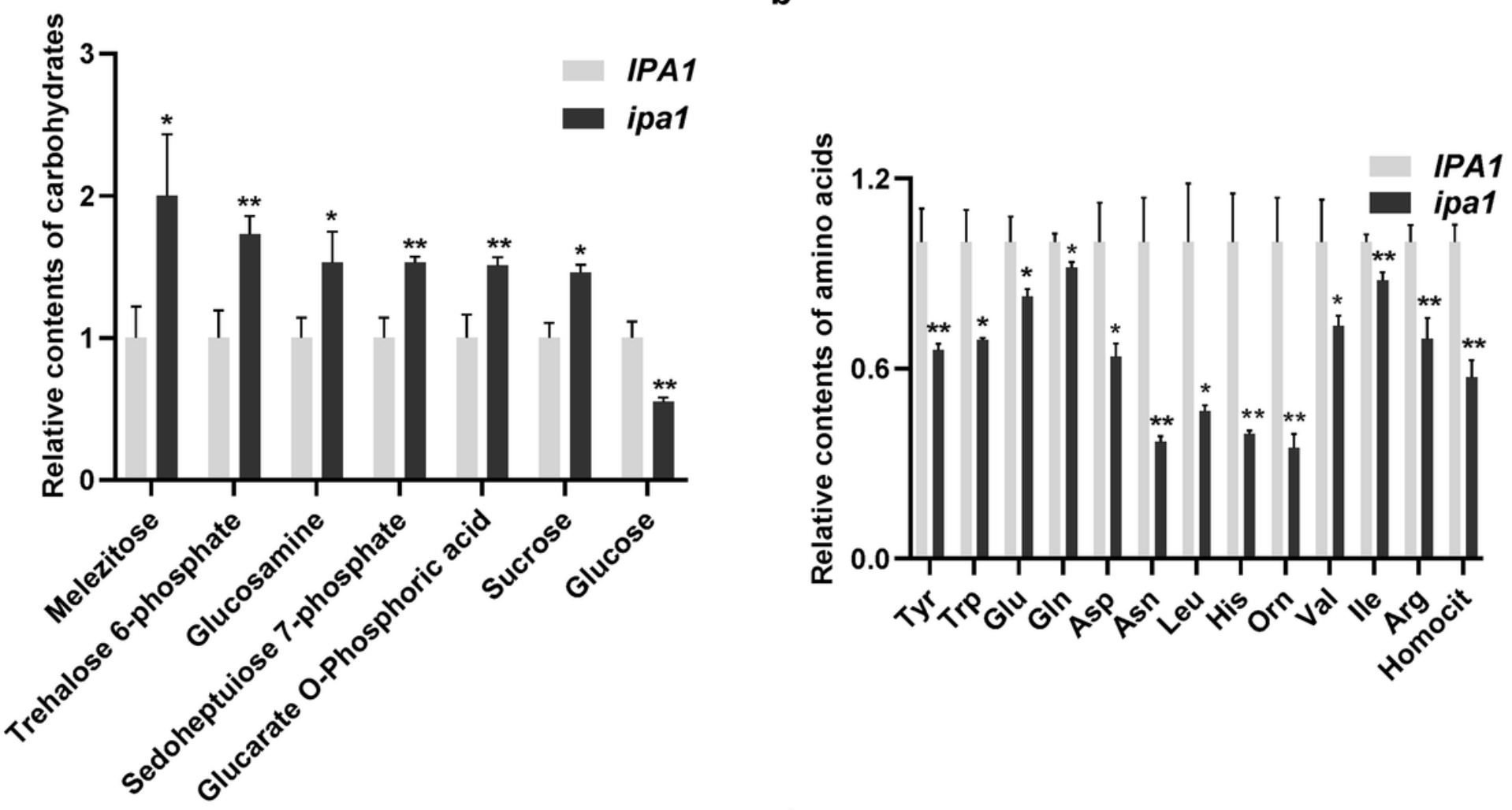

C
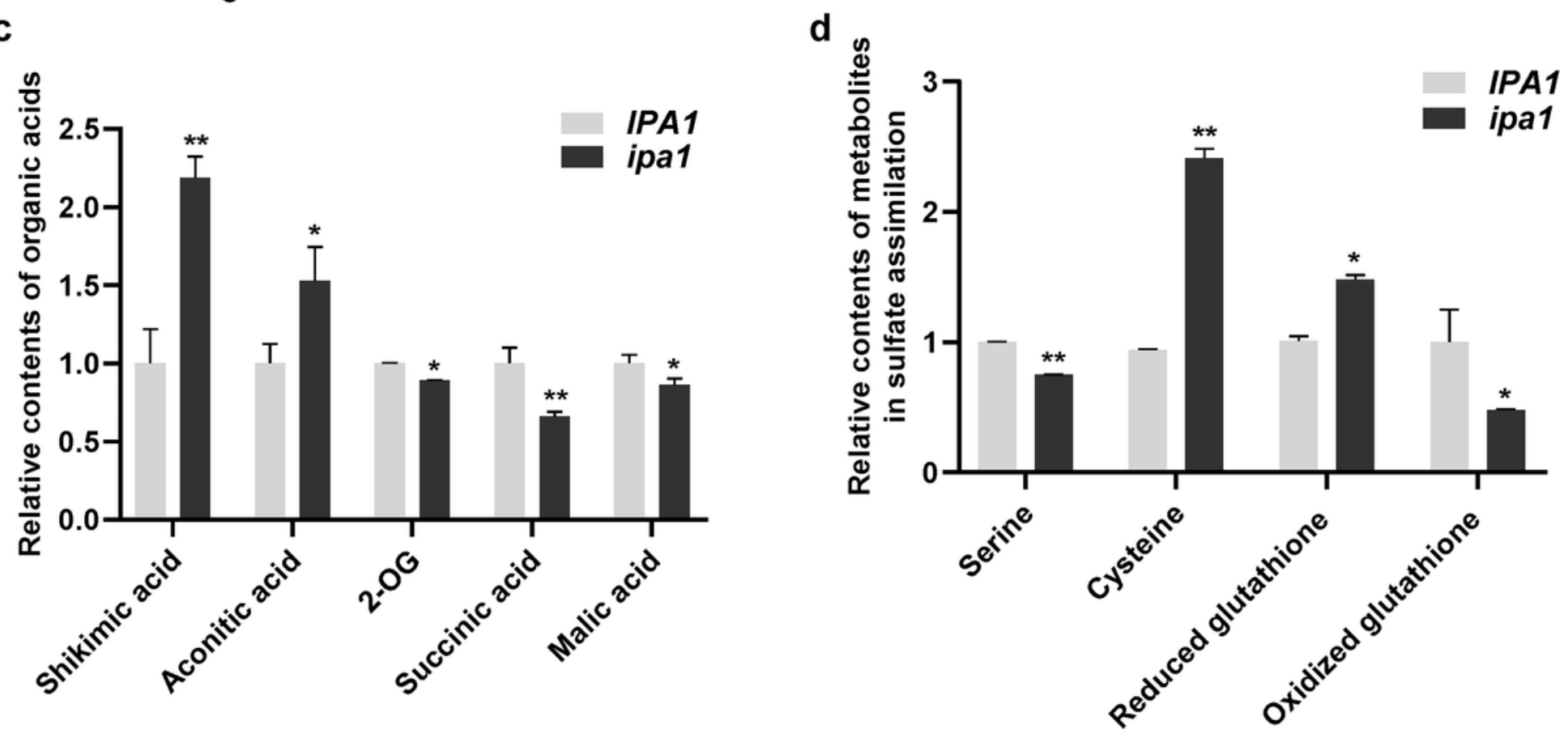

Figure 5

Contents of key metabolites in primary metabolism of the IPA1/ipa1 seedlings. (a-d) Contents of carbohydrates, amino acids, organic acids and metabolites in sulfate primary assimilation processes, respectively, in the IPA1/ipa1 seedlings. Data represent the means $\pm S E(n=3)$. *VIP $\geq 0.5, P<0.05$, t-test. ** $V I P \geq 0.5, P<0.01$, t-test. 
a

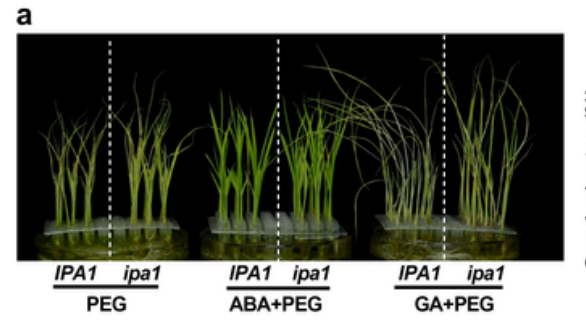

b
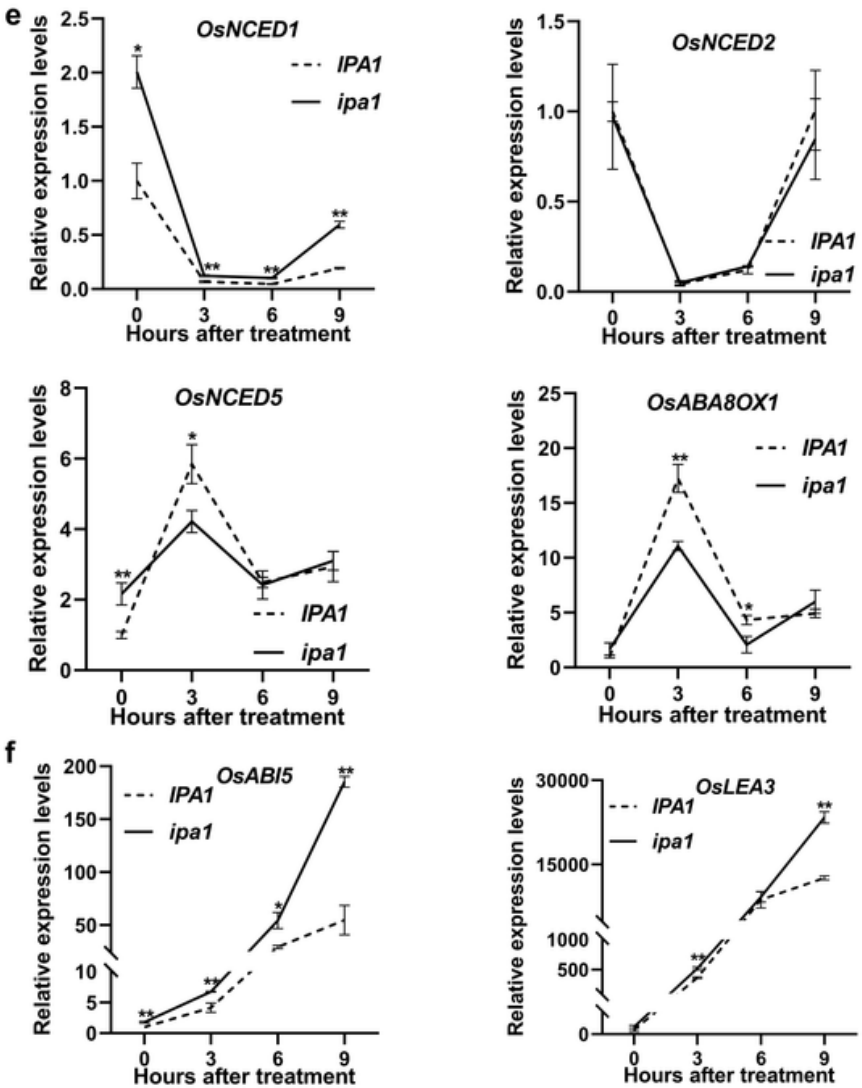

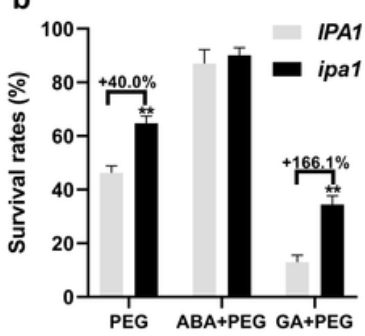

C
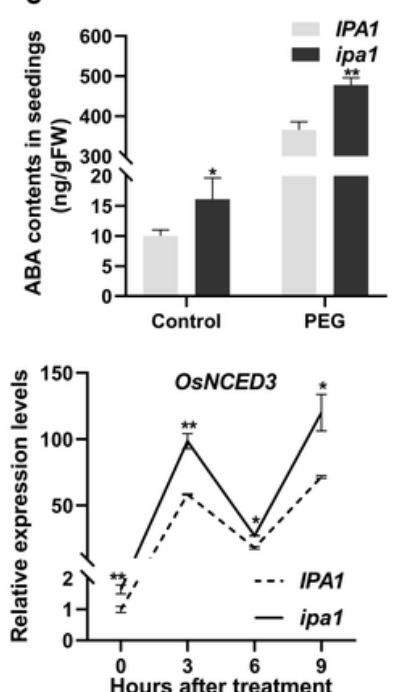
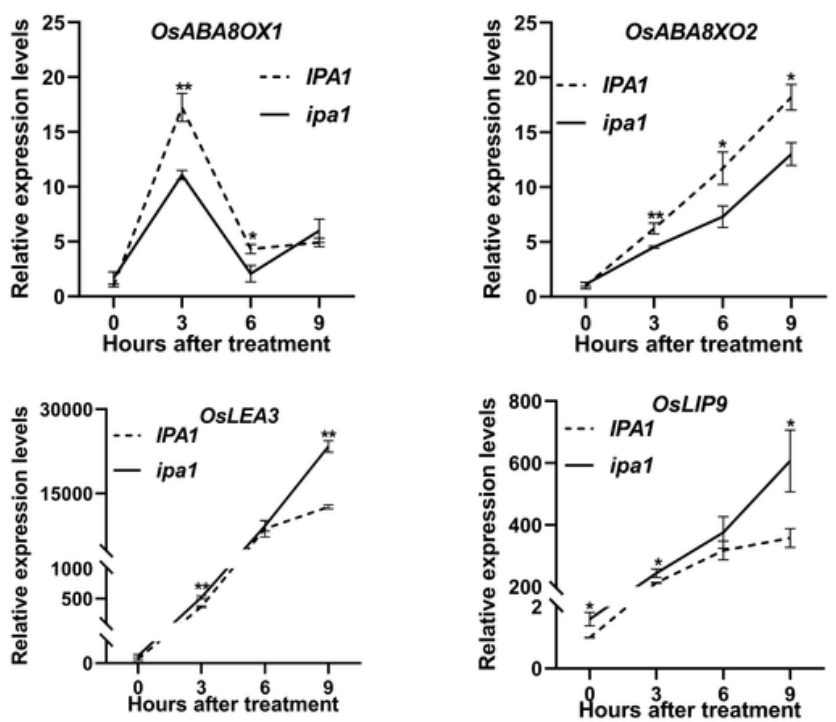
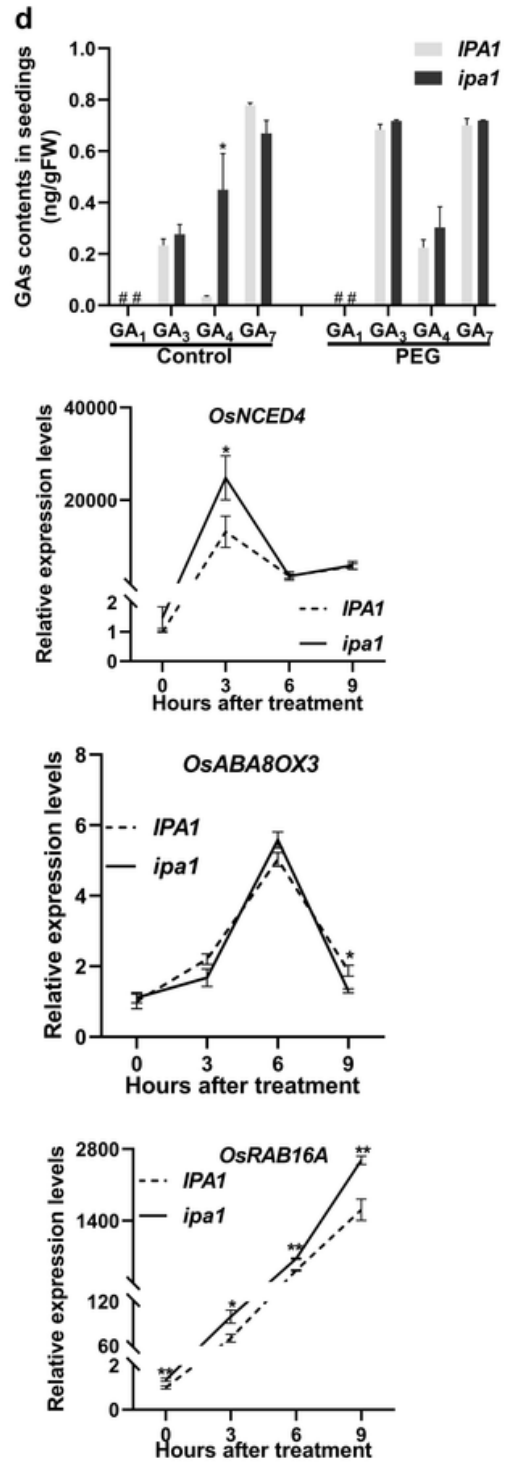

Figure 6

PEG tolerance response to exogenous $A B A$ and $G A 3$ treatments, endogenous $A B A$ and GAs contents of the IPA1/ipa1 seedlings and the expression of ABA-associated genes in the IPA1/ipa1 seedlings. (a) Phenotypes of the IPA1/ipa1 seedlings treated by ABA and GA3 with PEG. The IPA1/ipa1 seedlings cultured in normal condition were treated with ABA $(5 \mu \mathrm{mol})$ and GA3 $(10 \mu \mathrm{mol})$, respectively, then transmitted to culture solution with $25 \%$ PEG. (b) Survival rates of the IPA1/ipa1 seedlings in corresponding conditions. Each experiment has three replicates, and eighteen seedlings were tested in each replicate. (c, d) ABA and GAs contents in the IPA1/ipa1 seedlings, respectively. (e) The expression levels of genes involved in ABA biosynthesis and catabolism in the IPA1/ipa1 seedlings. (f) The expression levels of marker genes of the ABA pathway. Data represent the means $\pm S E$. ${ }^{*} P<0.05$, $t$-test. $\star * \mathrm{P}<0.01$, t-test. 
a

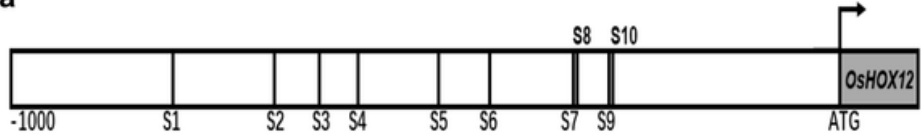

c

pGADT7+pHOX12-AbAi

IPA1 SBP $^{+} p H O X 12-\mathrm{AbAi}$
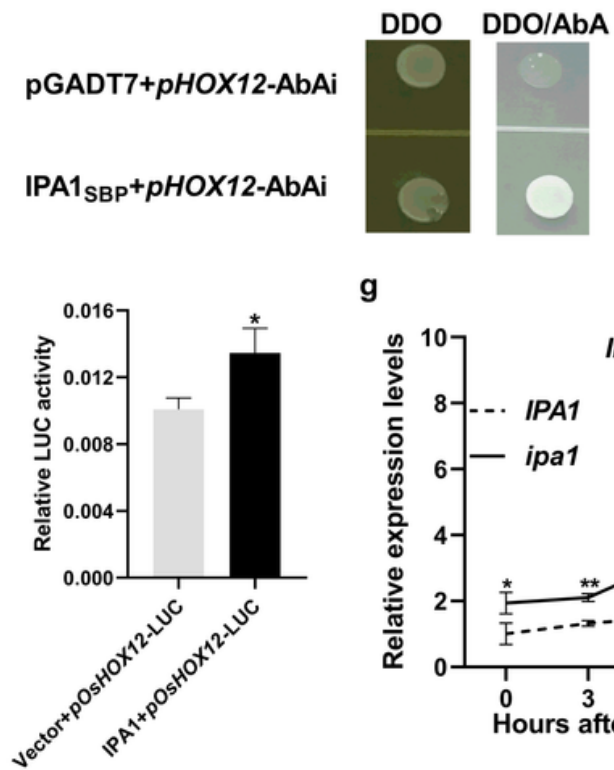

$\mathbf{f}$

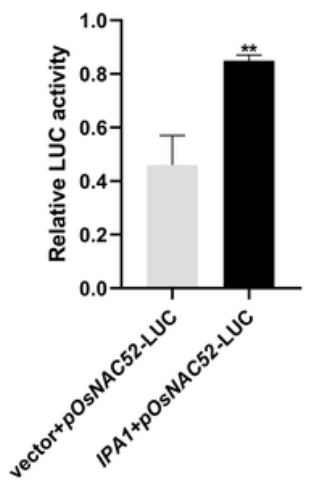

g
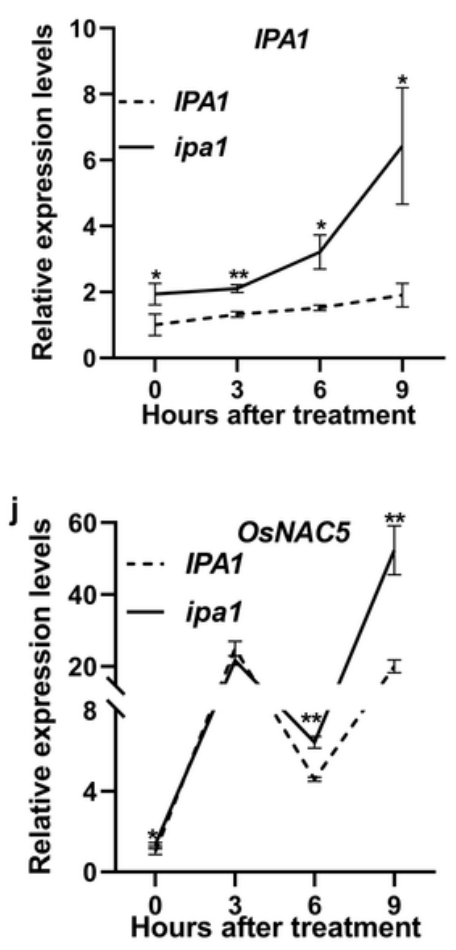

b

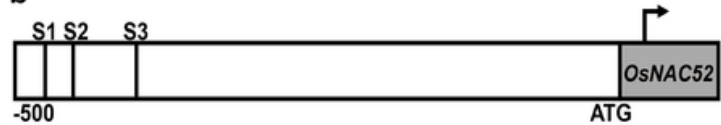

d

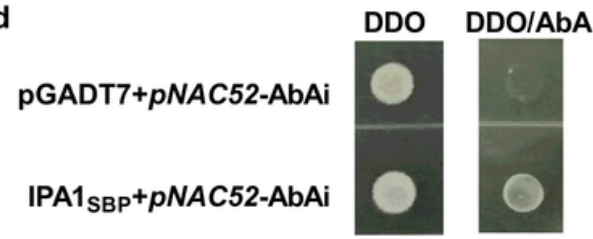

h

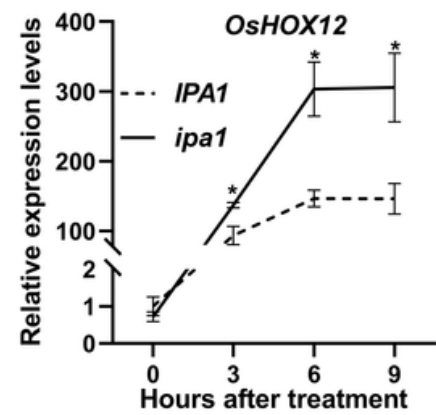

i

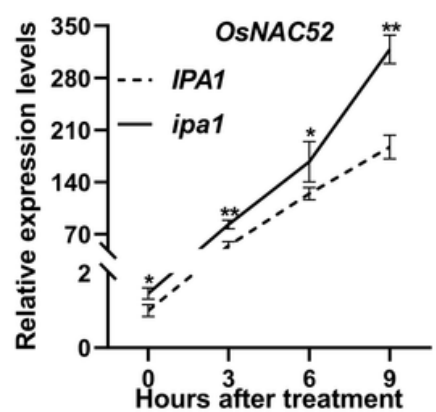

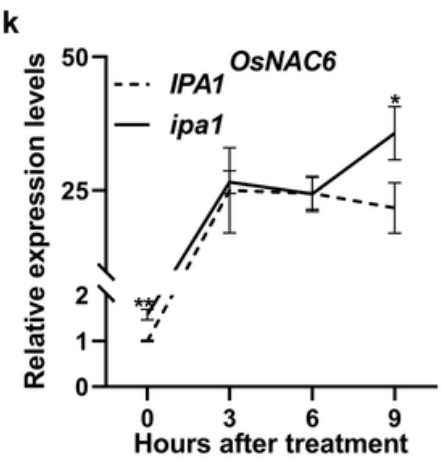

I

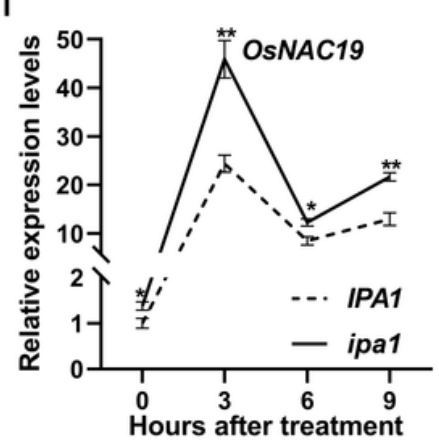

Figure 7

IPA1 directly activates the promoter activity of OsHOX12 and OsNAC52, and up-regulates the expression of the other NAC family genes. (a) Schematic of OsHOX12 promoter. S6 sequence is GTACGTACGTAC, and the other sites sequence is GTAC. (b) Yeast one-hybrid analysis with the fragment containing S1-S10 as bait. (c) Schematic of OsNAC52 promoter. The S1-S3 sequence is GTAC. (d) Yeast one-hybrid analysis with the fragment containing S1-S3 as bait. $(e, f)$ Relative LUC activity in transient transactivation assays. (g-l) Relative expression levels of IPA1, OsHOX12, OsNAC52, OsNAC5, OsNAC6 and OsNAC19, respectively. Data represent the means $\pm S E(n=3) .{ }^{*} P<0.05$, t-test. ${ }^{*} P<0.01$, t-test.

\section{Supplementary Files}

This is a list of supplementary files associated with this preprint. Click to download. 
- SupplementaryTable2.xIsx

- SupplementaryTable3.xlsx 